\title{
The Physical Essence of Time
}

\author{
Igor Maydykovskiy, Petras Užpelkis
}

\author{
«Biveja» JSC, Private Research Laboratory (Lithuania) \\ www.biveja.com e-mail: biveja@gmail.com
}

\begin{abstract}
The article considers the model of the space-frequency-time continuum, according to which the physical essence of Time is manifested as a fraction of electromagnetic energy spent on updating a material object in a cyclic process of copying-incarnation. For all structural levels of physical reality, the value of this fraction is a fundamental constant, which can be represented as the tangent of the loss angle, or expressed in radians, as the angle of inclination of the evolutionary spiral, which characterizes the rate of change of states or the duration of events and processes. The value of this constant can be calculated, and its value turns out to be identically equals to the square of the fine structure Constant $\left(\alpha^{2}\right)$. The description of the method for identifying a new constant allows us to present the formula of Scientific Discovery as the Physical Essence of Time.
\end{abstract}

Key words: physical essence of Time, constant of fine structure, 5th dimension evolutionary spiral, space-frequency-time continuum.

\section{Introduction}

In classical physics, there is no single generally accepted theory that explains and describes the physical essence of such a concept as time. According to the accepted paradigm, Time is an a priori characteristic of the world that is not definable in any way. As a measure for measurements, a certain, usually periodic sequence of events is used, which is recognized as the standard of a period of time. This principle is used in the design of watch movements and time measurement systems.

All processes in the world, regardless of their complexity, do not have any effect on the course of time; therefore, time in classical physics is called absolute. The absoluteness of time is mathematically expressed in the invariance (immutability) of the equations of Newtonian mechanics with respect to Galileo's transformations. That is, all moments in time in the past, present and future associated with the duration of events, processes, or a change of state are equal to each other. Time is uniform, and its movement (pace) remains unchanged at any point in space.

In accordance with the previously presented definitions of Space, Energy, Matter and Field [1], [2], [3] Time is a unifying physical parameter and without understanding its physical essence it is impossible to substantiate and conduct any physical experiment that could confirm the principles and provisions of the Theory of the phase medium. The physical models of Space, Energy, Matter and Field are based on the Holographic principle of organizing the structure of space and the physical mechanism of copying-incarnation, which are based on the system for generating the absolute scale of reference frequencies. In this sense, the Holographic Principle, by definition, requires the absolute stability of any parameters (including Time) participating in the formation of the wave matrix and its materialized projection in the form of physical objects and processes. It is the stability of the reference and structural frequencies at the program level that ultimately ensure the stability of the structures of the material world at the object level.

Let us recall the essence of these definitions, as they are presented in the Theory of the Phase Medium: 
- The space is a homogeneous, regular elastic structure or phase medium in the form of an infinite set of self-similar structural elements - quantum resonators.

- Physical reality is a materialized projection of the wave matrix in a cyclic process of copyincarnation. The structural unit of the wave matrix and material objects is a quantum of matter, consisting of a certain number of structural elements of the phase medium.

- Matter is a modified medium in the form of an elastic structure, consisting of a certain set of quantum resonators with different degrees of deformation of the elements of the phase medium and with different degrees of synchronization of oscillations.

- The Mass of a body can be represented as a measure that allows, in terms of the degree of synchronization of oscillations and the degree of deformation of the elements of the phase medium, to compare different levels of modification of the medium, linking these levels with different density of matter.

- The Energy of Space is concentrated: a) in the energy of elastic deformation of the structural elements of the phase medium, (according to the official paradigm, called dark energy); and b) in the energy of quanta of matter, which are in a state of continuous transformation, when electromagnetic energy, the shell of a quantum of matter in the form of a vortex structure periodically takes the place of the nucleus and transforms into the energy of longitudinal spherical waves, that is, into dark energy, and then again becomes a shell.

- The Field, regardless of its nature, is the structure of the density gradient distribution in the form of areas of compression - tension in the structure of an elastic medium. The structure of the field (for example, magnetic) repeats the interference (holographic) structure of spatial frequencies in the local area of space.

\section{About the Theory of Relativity}

The General Theory of Relativity proposed by Einstein (1905), which is based on the postulate of the relativity of time, cannot be considered a theory that can bring us closer to understanding the physical essence of Time, since, having the status of an official theory, practically closes the possibility of a scientific discussion on this topic. In spite of this recently, many critical articles have appeared that prove the abstractness and inconsistency of the Theory of Relativity, in the absence of any practical expediency in its application. To understand to what extent the criticism of the Theory of Relativity corresponds to reality, let us dwell on three out of ten postulates on which Einstein's theory is based and which are directly related to the concept of Time.

The first postulate says that the simultaneity of events spaced apart in space is relative. In accordance with this postulate, an observer, comparing the readings of two clocks separated in space, can do this in one single way, using for this purpose a communication channel with an information transmission rate limited by the velocity of light.

- What is the error here?

An error in the substitution of the concept - Time, - the concept - the observed clock readings.

Obviously, the value of the speed with which the information about the clock reading is delivered to the observer cannot be the reason for the change in the course of Time at the points of registration of the events themselves. 
In this sense, this postulate violates the basic law of physics - the principle of causality. An important consequence of this postulate is the statement that if absolute simultaneity of events is impossible, and then absolute time cannot exist, which is equally current in all reference frames.

Summarizing, we can state that the considered postulate contradicts one of the fundamental provisions of classical physics, according to which the properties of physical bodies and processes cannot depend on the reference frame, observation methods and methods of recording physical parameters. Moreover, the relativity of time, according to Einstein's theory, makes it impossible for the existence of a system for generating an absolute scale of reference frequencies, without which, according to the Theory of the phase medium, the physical mechanism of copying-incarnation cannot exist.

The second postulate speaks of the invariance (immutability) of the time interval when information about an event arrives using the same information transmission channel, but the object itself is moving. According to the formula proposed by Einstein, real time ( $\left.\Delta \boldsymbol{t}_{\text {real }}\right)$ for an object moving at a speed $(v)$ close to the velocity of light $\left(c^{*}\right)$ supposedly tends to infinity:

$$
\Delta t_{\text {real }}=\Delta t / \sqrt{1-\frac{v^{2}}{c^{2}}} ; \text { (сек) }
$$

Here, as in the case with the assessment of the simultaneity of events spaced apart in space, information about the clock reading cannot be the cause of a change in the course of time on the object itself, even if the object moves away at a speed close to the velocity of light. After all, the deceleration of the clock is obvious only to the observer, while the clock readings on the object, like the object time itself, do not change. It is about the same as if the inspector, measuring the speed of a retreating car, claimed that Time inside your car slowed down, because the Doppler Effect causes the hands of a car clock to move slower in its view.

According to the presented model [2], the object (including the clock) is updated with a frequency: $v_{r b}=v_{e}=2,4892 \cdot 10^{20}(\mathrm{~Hz})$ at the rate of the process itself $\left(\boldsymbol{c}^{2}\right)$. That is, taking into account the quantum nature of the renewal process, both of the object itself and its wave matrix, the object is not threatened with a reduction in size, or an increase in its mass, or a stop of the processes that determine its physical essence.

It should be noted that the above formula cannot be substantiated from the point of view of any physical model. Since any physical process or object will be destroyed if we assume the instability of the structural frequencies of the harmonic series, which at the program level of physical reality is the basis for the formation of the process itself. That is, this formula, reflecting, in fact, the abstractness of the proposed mathematical model, cannot be the basis for a transparent physical model.

To finally be convinced of the inconsistency of the above formula, we repeat the thought experiment with an object that moves with about velocity of light. To do this, we place the observer in the center, around which the object moves in a circular orbit.

This can be, for example, a spacecraft that revolves around the Earth at a speed close to the velocity of light. In this case, making one revolution, the object sends a short pulse of light to the Earth. Let the time interval between light pulses be of the order of: $\Delta \boldsymbol{t}=20 \mathrm{~ms}$, and its duration: $\boldsymbol{\tau}$ - no more than $30 \mathrm{fs}$. To eliminate the influence of the Doppler Effect, it is possible to install a pulse generator on a conventional stationary satellite and activate it at the time of the passage of the spacecraft. With such an experiment scheme for an observer on Earth in his frame of reference, the apparatus seems to be standing still, having turned into a "point". 
Obviously, both recorders will show the same time. In this case, the synchronous course of the clock will be confirmed by the constancy and equality of the time intervals between the pulses. As for the phase delay associated with the limitation of the signal propagation speed, at the point of observation its influence can in no way affect the value of the interval between pulses, and therefore the "mathematical hypnosis" of Einstein's formula does not work here. Therefore, no matter how much "spinning" around the Earth with about velocity of light, the "paradox of twins" will not happen, and after leaving the spacecraft, the astronaut will not look younger than his peers will.

Thus, our thought experiment proves that as soon as we pass from a linear coordinate system to a spherical one, the "charm" of Einstein's theory and formulas disappears.

The third postulate, which is worth paying attention to, says that space has physical properties. That is, so that Time could be considered one of the coordinates of a single 4-dimensional space-time, Einstein actually endowed space with physical properties, filling the "empty" space with the energy of gravitational waves. Einstein believed that "... the environment of space is deformed (bent) under the action of the masses of matter", that is, under the action of gravitational forces. In this case, the interaction of material objects or processes is carried out through the field, which is created by the objects themselves. The field gradient depends on body mass and is an indicator of the curvature of space. That is, in Einstein's view, matter forms the structure of the gravitational field, while the medium remains a passive component of space.

In such a situation, when matter controls space, by means of a field, in the form of a "special kind of matter", that is, when cause and effect are reversed, it is impossible to create a unified field theory exactly the same theory that Einstein developed all his life. The General Theory of Relativity in this sense was just an attempt to find a connection between Time and space, using the dependence of the spatial component of the metric on time through the field equations proposed by Einstein. At the same time, the connection between Time and space could be indirectly established through the properties of impulse and energy. Since the change in momentum, being a time characteristic of the force, is determined by its momentum: $\boldsymbol{F} \cdot \Delta t$, provided that space is isotropic (symmetry and uniformity), and the change in energy is determined by the spatial characteristic of the force - its work: $\boldsymbol{F} \cdot \Delta \boldsymbol{r}$, under a similar condition of uniformity of Time.

It is obvious that if, according to the special theory of relativity, the possibility of slowing down or accelerating Time is admitted, then both conditions cannot be fulfilled simultaneously, or otherwise the law of conservation of Energy will be violated.

Thus, the analysis of the postulates of the General Theory of Relativity and the presented conclusions make it possible to exclude most of the provisions of this theory when considering the processes of development and interaction of structures and objects within the framework of the Theory of the phase environment.

Distortions in the representation of physical reality, which became a consequence of Einstein's delusions, influenced many directions in physics, therefore it is very likely that the truth in understanding and disclosing the physical essence of Time can radically change the prevailing concept not only of the space-time continuum, which at the moment is exclusively a product of the General Theory of Relativity.

In this sense, critical remarks about Einstein's Theory and the principles and definitions proposed in this article regarding the physical essence of Time are a proposal for a paradigm shift and for the formation of a new approach to understanding the laws of Nature and the structure of the Universe. 


\section{Space-Frequency-Time Continuum}

One should not think that when a scientist-physicist utters the words: "space-time continuum" he fully understands the meaning of this concept and presents a physical model that allows to unite space and Time. As already mentioned, initially, Einstein's field equations were only needed to represent the scale factor of the universe as a function of time. Subsequently (1927), the FLRW (Friedmann - Lemaître - Robertson - Walker metric) metric was, within the framework of the General Theory of Relativity, an exact solution of Einstein's field equations, and made it possible to describe a homogeneous, isotropic, expanding (or otherwise contracting) Universe, which is linearly connected and can be multidimensional [5].

The concept of an expanding Universe was directly related to the inflationary theory of the Big Bang, which, as you know, was born at the tip of a pen (1922), when a mathematician from Russia A.A. Friedman, analyzing the equations of Einstein's General Theory of Relativity, came to the conclusion that the Universe is unsteady, and its curvature changes. In this case, the very solution proposed by Friedman opened up two possibilities of evolution - monotonic continuous expansion (contraction) or periodic increase and decrease in curvature. In the second case, the physical model of the Universe can be compared with a pulsating structure filled with energy in the form of a sphere, which periodically expands and contracts. The idea of an expanding Universe was supported by the American astronomer Edwin Hubble and the Russian scientist Georgy Gamov. As a result, the scientific community at that time considered the inflationary theory a more promising option.

A famous physicist, Roger Penrose decided to continue research in this direction (1965). Moreover, today (2020), already being a Nobel Prize winner, he declared that the evolutionary process of the development of the Universe can be cyclical. In this case, Penrose is actually trying, without violating the canons of the official paradigm, to combine both versions of the solution to the equation of General Relativity, combining the mathematical apparatus of quantum mechanics with an abstract model of General Relativity.

As you know, the term "collapse of the wave function", used in quantum mechanics, is a purely mathematical device used to solve the Schrödinger equation. The Schrödinger equation describes, using the de Broglie probability wave, the probability of finding a particle in a particular state at a given point in space and can be represented in expanded form as:

$$
\hbar \cdot \frac{c^{2}}{\omega}(\operatorname{div} \operatorname{grad}) \cdot \Psi(x, y, z) \cdot \varphi(t)+i \cdot \hbar \cdot \frac{\partial}{\partial t}=U \cdot \Psi(x, y, z) \cdot \varphi(t)
$$

Here, $\boldsymbol{\Psi}=\Psi(x, y, z) \cdot \varphi(t)$ - is the wave function, presented as a time-dependent oscillator; $\boldsymbol{\Delta}=$ div grad - is the Laplace operator; $\boldsymbol{\hbar}$ - Planck's constant; $\mathrm{m}$ is the rest mass of the particles; $\boldsymbol{U}$ - is potential energy; $\boldsymbol{i}=\sqrt{-1}$.

Without proposing a physical mechanism for the mutual transformation of the wave process into a material form in the form of a particle, Penrose believes that at a certain value of mass ( $\boldsymbol{m}$ ) (approximately equal to the mass of Planck's substance), the wave function $(\boldsymbol{\Psi})$, being in a superposition between quantum states, cannot to maintain its existence within the limits of the energy difference corresponding to this mass. In other words, according to Penrose, a quantum state is in a superposition until the curvature of space-time reaches a significant level and causes a collapse of the wave function. Then the system can again go into an indefinite state of singularity. 
In this case, the space can collapse again to the size of a pea and after a new Big Bang, the process will repeat itself. Thus, with the light hand of Penrose, the Schrödinger equation, as it were, united quantum mechanics and cosmology.

The assumption put forward by Penrose can be critically evaluated from the point of view of the provisions of the Theory of a phase medium. The first thing that should be paid attention to is the absence of reference to the physical mechanism due to which the transformation of a wave into a particle is carried out. Indeed, in fact, the Schrödinger equation does not describe a physical process, and the distribution of energies here has a different meaning than the one that Penrose is trying to introduce. The ratio of energies indicates only the value of the probability of finding a particle in a particular state. Moreover, the fact of "materialization" of a particle, as if recorded with the help of a detector, is associated only with the very fact of registration, and not with internal or external processes that form the structure of a particle in the form of a material object.

That is, in this case, we can say with confidence that the fact of registration of a particle only confirms its next incarnation as an event that was registered with the help of a detector. Obviously, if one wave process is recorded using a detector, the operating principle of which is based on another analogous wave process and both processes, having a quantum nature, are not related to each other except for the very moment of registration, then the registration process will be probabilistic. This is confirmed by the Schrödinger equation, which was previously presented as the de Broglie probability wave.

Now, if we try at the level of physical models to combine the process of expansion of the Universe, after the alleged Big Bang, with a cyclic process of increasing and decreasing curvature, then from the point of view of the physical mechanisms regulating the cyclic process, preparation for a new Big Bang can be considered only against the background of the phase of "compression", but not against the background of the "expansion" phase.

But even if we accept the idea of "collapse of the wave function" and assume that in the process of expansion of the Universe, it will have to go back to the state of singularity, then the cyclic process associated with the reversible transformation of dark energy into energy of matter, being the basis of the existence of the Universe, inevitably should will collapse. That, for obvious reasons, cannot happen, since the energy of elastic deformation of the medium cannot disappear, leaving behind a absolute emptiness and destroying the harmonic process due to which it exists.

If we follow the postulates of Einstein's theory of relativity, the theory of propagation of EM - waves of Maxwell and the inflationary theory of the expanding Universe, then a logical question arises: What form of Energy fills empty space? Maxwell, endowing space with physical properties, "secretly" believed that the emptiness is filled with ether. Hence, appearing in the literal sense of the emptiness, the absolute dielectric constant $\left(\boldsymbol{\varepsilon}_{\boldsymbol{0}}\right)$ and the absolute magnetic permeability of the vacuum $\left(\mu_{0}\right)$.

For Einstein, this was the potential energy of gravitational waves, as well as an unknown form of energy, later called dark energy, which supposedly causes the universe to expand. Moreover, none of the "founders" of emptiness thought about the fact that no matter what name you give, Energy is, first of all, a measure of transformation of one form of motion of matter into another form of its motion. 
And in this sense, according to the authors, one should not look for a "common denominator" for all the named indefinite forms of energy, if within the framework of a physical model based on the holographic principle of organizing the structure of space, Energy is defined as the deformation energy of the structural elements of the phase medium. This form of energy, presented in the official paradigm as dark energy, is concentrated in the energy of the reference frequencies of spherical longitudinal waves and exists due to the reversible continuous process of transformation of the deformation energy of the structural elements of the phase medium into the electromagnetic energy of matter [2]. The energy distribution of the reference frequencies obeys the fractal principle, and the frequencies themselves are represented by the zero row of the matrix (formula (12-2).

The values of all frequencies are expressed in $\mathrm{Hz}$, while each frequency range begins with a reference frequency: $\tilde{\boldsymbol{v}}_{\boldsymbol{0}}(j=0,1,2 . ., \boldsymbol{u})$, is built on the basis of a harmonic series in accordance with the octave principle: $\tilde{\boldsymbol{v}}_{01}=2 \cdot \tilde{\boldsymbol{v}}_{00}, \tilde{\boldsymbol{v}}_{02}=2 \cdot \tilde{\mathbf{v}}_{01}, \ldots \tilde{\boldsymbol{v}}_{0 u}=2 \cdot \tilde{\boldsymbol{v}}_{0}(u-1)$.

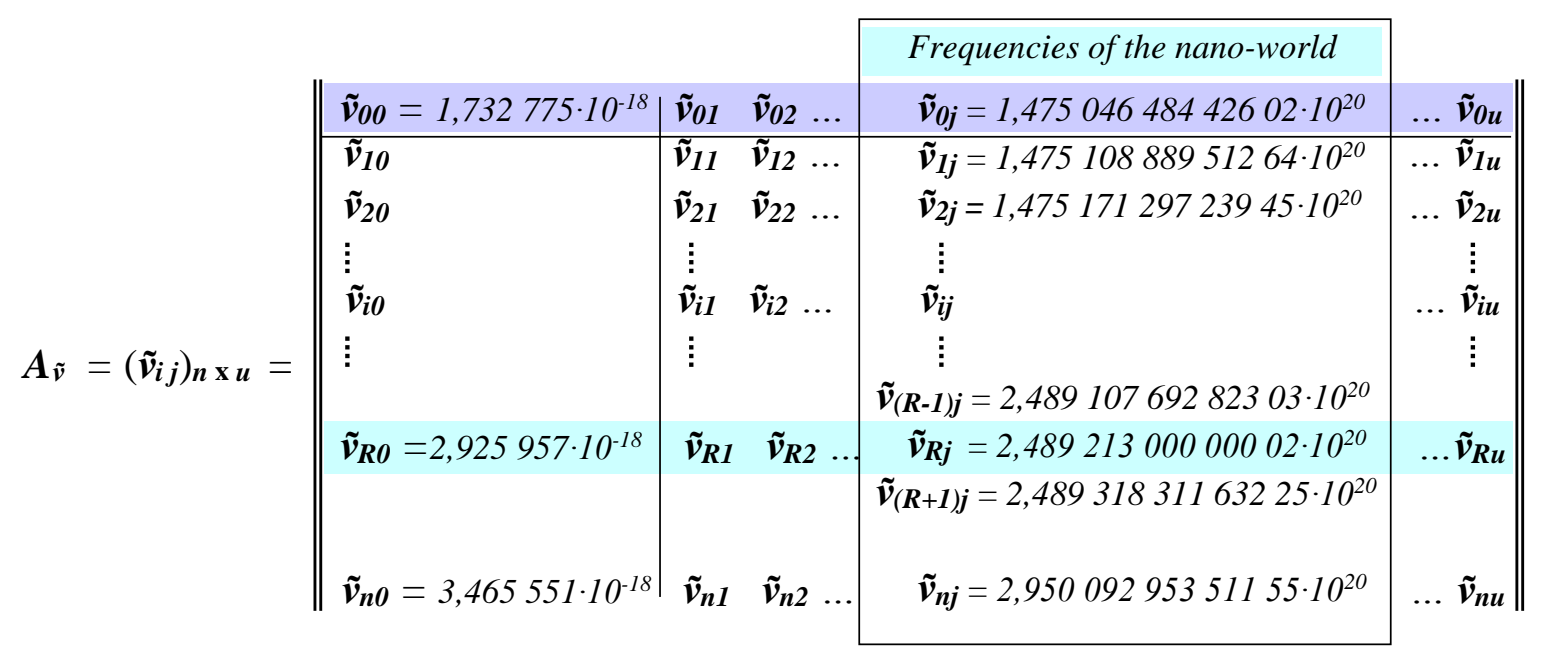

The total Energy of the zero-line harmonics is the dark Energy, which can be defined as the value of the spectral density of Energy in accordance with the generalized Rayleigh formula [2]. In each of the spectral ranges, this energy in the cyclic process of copying-incarnation is transformed into the electromagnetic energy of matter at all structural levels of physical Reality, from the scale of the Universe to the range of an energy quantum.

The presented matrix: $\boldsymbol{A} \tilde{\boldsymbol{v}}=\left(\tilde{\boldsymbol{v}}_{i}\right)_{n} \times \boldsymbol{u}$ serves for the formal description of the geometric field of spatial frequencies or the Hologram of the Universe, as well as for the representation and analysis of frequency ranges of all scale levels, starting with the quanta of matter and ending with the period of the evolutionary cycle of the Universe. In this case, as previously established [2], the total number of spectral ranges corresponds to the number of scale levels and is equal to, $\boldsymbol{u}^{*}=216$.

The method for calculating the number of structural frequencies in each range will be presented later, here we will use the earlier calculations and consider two spectral range is the ultra-low frequency range and the copying-incarnation frequency range.

The first of them - the frequency range $\left(\begin{array}{llllll}\tilde{v}_{00} & \ldots & \tilde{v}_{i 0} & \ldots & \tilde{v}_{n 0}\end{array}\right)$ - determines the size, structure and renewal period of the Universe. This period is in the same harmonic series with the refresh rates of other scale levels. The period value corresponds to the evolutionary Cycle:

$$
\boldsymbol{T}_{\text {Real }(1)}=1 / \tilde{v}_{\text {Real }}=10,84 \text { bill. } \text { Years }
$$


Accordingly, the period of the zero harmonic, which determines the dimensions of the sphere of the Universe, will be equal to $\boldsymbol{T}_{(0)}=1 / \tilde{\boldsymbol{v}}_{00}=18,30$ bill.Years, and the period of the 2nd harmonic, which determines the value of the Energy of space: $\boldsymbol{T}_{(\boldsymbol{n})}=1 / \tilde{\boldsymbol{v}}_{\boldsymbol{n} \boldsymbol{0}}=\mathbf{9 , 1 5}$ bill.Years. The value of this energy was estimated at about $74-75 \%$ of the total energy of space, and it was this part of dark energy that was measured by indirect methods. The energy of the 2 nd harmonic is transformed into the energy of electromagnetic oscillations of the material World as a result of the continuous process of copyingincarnation.

The time of existence of the Universe according to data from different sources, taking into account the error, is in the range of 11.5-13.8 billion years. From which it follows that our Universe is at the present time, at least at the beginning of the 2 nd evolutionary Cycle or in numerical form:

$$
\boldsymbol{T}_{\text {Real }}=\boldsymbol{T}_{\boldsymbol{R}(1)}+\Delta \boldsymbol{T}_{\boldsymbol{R}(2)}=\mathbf{1 0 , 8 4}+(1,8 \pm 1,1) \text { bill. Years }
$$

The second $\mathrm{j}$-th spectral range $\left(\tilde{\boldsymbol{v}}_{0 j} \ldots \tilde{v}_{i j} \ldots \tilde{\boldsymbol{v}}_{\boldsymbol{n}}\right)$, allocated in the matrix: $\boldsymbol{A}_{\tilde{\boldsymbol{v}}}=\left(\tilde{\boldsymbol{v}}_{\boldsymbol{i}}\right)_{\boldsymbol{n}} \times \boldsymbol{u}$, corresponds to the range of structural frequencies, among which there is a reference frequency of copyingincarnation - $\tilde{\boldsymbol{v}}_{\boldsymbol{R} j}$. The value of this frequency: $\tilde{\boldsymbol{v}}_{\boldsymbol{R}}=2,489213 \cdot 10^{20}(\mathrm{~Hz})$ corresponds to the frequency of the electron's incarnation.

The distribution of structural frequencies within each frequency range is subject to a tempered harmonic series. Taking as a basis the frequency of the electron incarnation, we can calculate the values of the frequencies of the entire tempered series in the selected spectral range. This series will correspond to one of 216 spectral ranges in the grid of allowed frequencies, which in the form of the structure of the geometric field of spatial frequencies (Hologram of the Universe) was formed at the software level as a result of the functioning of the fractal cyclic iterative algorithm with one or more components of the random harmonic series turned off [2].

In order to be able to directly determine the number of permitted frequencies $(\boldsymbol{n})$ as a result of our research, it is necessary to understand the principle of structural organization inherent in the formation mechanism of the tempered harmonic series, as well as to analyze the conditions and constraints that were included in the cyclic iterative algorithm at the formation stage "geometric field of spatial frequencies". The result of the planned research should be a theoretical justification and a physical model that allow us to understand the physical essence of Time.

\section{The physical essence of Time - theory and experiment}

According to the previously proposed postulate [2], Space is a uniform regular elastic structure that does not allow emptiness. A similar condition can be extended to the frequency scale, which, taking into account the value of the minimum energy quantum, also does not allow the existence of "holes" in any of its spectral ranges, and the total number of frequencies circulating in Space will in this case be determined only by the Q-factor of resonant systems that determine the structure physical objects and processes. Therefore, any harmonic sequence, being built, by implementing the algorithm of a cyclic iterative structure with switching off one or more components of a random harmonic series, will have the right to exist.

From the above, an important conclusion follows, which radically changes our ideas about the structure of the Universe and space - space is discrete, and the number of nested subspaces simultaneously determines the number of nested Holograms. 
Each subspace has its own harmonic series, based on mutually exclusive frequencies, which ensures their independent existence. Thus, accepting the holographic principle of organizing the structure of space and the fractal principle of the distribution of Energy, it is necessary to consider the physical model of space as a Space-Frequency-Time Continuum.

Figuratively we can say that the most important "experiment" that still has to be carried out by mankind has already been carried out by Nature itself, and we can only understand the meaning of the "obtained results". The first step in this direction can be considered the representation of the frequency domain and the presence of reference and structural frequencies in space, not only as an energetic and structural basis of matter, but also as the 5th dimension. Then the concept of: SpaceFrequency-Time Continuum will acquire real physical meaning.

At the same time, for further research, it is necessary to introduce a new concept - the frequency horizon and the corresponding designation for this concept: $\boldsymbol{H}_{\boldsymbol{F}}$. This concept, reflecting the physical essence of the wave matrix: $\boldsymbol{A} \tilde{\boldsymbol{v}}=\left(\tilde{\boldsymbol{v}}_{i j}\right)_{\boldsymbol{n}} \times \boldsymbol{u}$, develops the previously proposed definition of space and allows us to formulate the Postulate on nested subspaces.

Postulate: Space is discrete and structurally consists of a set of equal wave matrices. Each wave matrix in the form of a geometric field of spatial frequencies is constructed in relation to the rest of the matrices on the basis of the principle of mutually exclusive harmonic series. The formation of quantum space and its division into subspaces occurs according to the frequency principle: one Frequency of copying-incarnation, - one Wave matrix, - one frequency Horizon, - one space-time Quantum.

The 5th dimension in this interpretation can be graphically represented as a set of tensors (wave matrices), placed sequentially along the evolutionary spiral (Fig. 2), but structurally representing a single continuum

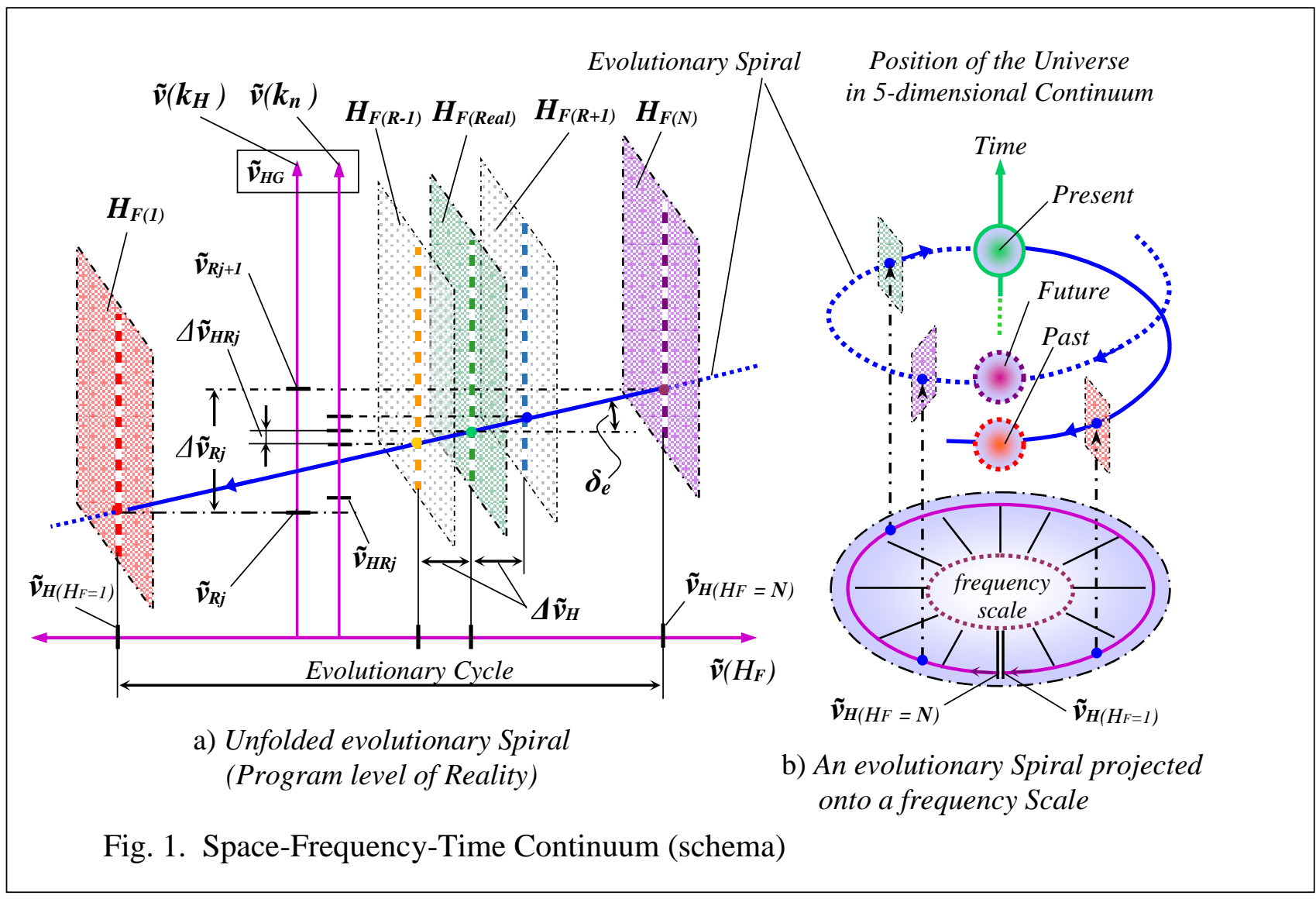


On the diagram: $\tilde{\boldsymbol{v}}_{\boldsymbol{H} \boldsymbol{G}}$ - general spatial frequency scale;

$\Delta \tilde{\boldsymbol{v}}_{R}$ - frequency band allocated for copying-incarnation frequencies in the 5 th dimension with reference to the wave matrix of physical reality;

$\tilde{\boldsymbol{v}}_{\boldsymbol{R} j}$ - frequency of incarnation of an electron in the frequency horizon of physical reality; $\tilde{\boldsymbol{v}}\left(H_{\boldsymbol{F}}\right)$ - scale of frequencies of the incarnation of an electron in the 5 th dimension (one frequency - one wave matrix - one subspace - one quantum of space - Time;

$\Delta \tilde{v}_{\boldsymbol{H}}$ - spectral bandwidth that separates copying-incarnation frequencies belonging to wave matrices of different frequency horizons;

$\boldsymbol{\delta}$ - angle characterizing the amount of losses in the cycle of modification of the wave matrix.

The space-Frequency-Time Continuum is presented in the coordinates of the frequencies of the 5th dimension, where the vertical is "the common frequencies" scale - $\tilde{v}_{H G}$; horizontally is "the space-time quanta" (nested subspaces) scale - $\tilde{\boldsymbol{v}}\left(\boldsymbol{H}_{\boldsymbol{F}}\right)$.

The scale of space-time quanta is presented in the form of a set of spectral band-pass filters, each of which corresponds to its own sequence of harmonic series, based on mutually exclusive frequencies. In the structure of space-time quanta (nested holograms), each spectral range of the wave matrix begins with a reference energy-carrying frequency, which is included in an element of a harmonic series built on the basis of the octave principle: $\tilde{\boldsymbol{v}}_{\boldsymbol{0} 1}=2 \cdot \tilde{\boldsymbol{v}}_{\boldsymbol{0}}, \tilde{\boldsymbol{v}}_{\boldsymbol{0} 2}=2 \cdot \tilde{\boldsymbol{v}}_{\boldsymbol{0} 1}, \ldots \tilde{\boldsymbol{v}}_{\boldsymbol{0} \boldsymbol{u}}=2 \cdot \tilde{\boldsymbol{v}}_{\boldsymbol{0}(\boldsymbol{u}-\boldsymbol{1})}$. These frequencies in the form of a grid of standing longitudinal spherical waves form the structural and energy basis of the Space-Frequency-Time Continuum in the form of nested holograms.

The placement of wave matrices presented in the diagram allows us to represent the process of changing the states of an object in a chain of events: past - future in a different way. If we assume that a new state of any object comes from the Future, replacing the Present, which then becomes the Past, then the change of states, can be associated with the continuous replacement of the holographic structure of the wave matrix in a cyclic process of a copying-incarnation.

In other words, the events themselves "move" along the evolutionary spiral from the Future to the Past in the form of a changing holographic structure, while maintaining the position of the Universe as an object in a 5-dimensional continuum (Fig. 1b). That is, unlike the official paradigm, this process should be associated not with the "movement" of the Universe from the Present to the unknown Future along the virtual (indefinite) axis of Time, but with continuous modification of the wave matrix of an object or a process in the frequency domain and its replacement with a "clone" from the Future followed by materialization in the time domain. That with the constancy of the period of the process, copying-incarnation will reflect the state of the physical World at a given moment in Time.

From an energy point of view, the process of modifying the structure of an object does not depend on the scale level, and the change of energy states occurs with the frequency of the electron incarnation, simultaneously at all levels, starting from the level of the energy quantum in the structure of the wave matrix. Therefore, we can talk about the absolute frequency synchronization of the process of modifying the wave matrix and the process of materialization of the object. That is, the process of changing the state of a 4-dimensional object is synchronized with the process of changing the state of the same object in the 5th dimension. 
In this case, the frequency of the electron materialization process $\left(\boldsymbol{v}_{\boldsymbol{e}}\right)$ in the copying-incarnation cycle will be limited in physical reality by the inertial properties of the medium, that is, by the limiting speed $c^{*}$. At the same time, the process of updating the two-dimensional wave matrix of an electron proceeding with a frequency $\boldsymbol{v}_{\boldsymbol{e}} \approx \boldsymbol{v}_{\boldsymbol{e}} \cdot 2,997 \cdot 10^{8}(\mathrm{~Hz})$, will be limited by the speed $\boldsymbol{c}^{2}$ [2]. Accordingly, while at the object level of physical reality in the time domain the process of renewal of the electron proceeds, at the program level in the frequency domain the wave matrix will be updated $2,997 \cdot 10^{8}$ times. Then, taking into account the frequency ratio, the total number of structural frequencies $(\boldsymbol{n})$ cannot exceed $\boldsymbol{c}^{*}$, which makes it possible to estimate the possible number of allowed structural frequencies in each spectral range as follows:

$$
\boldsymbol{n} \leq 2,997 \cdot 10^{8}
$$

In this case, in order to calculate the frequencies of the tempered harmonic series and the value of the width of the spectral bands for each of the frequencies, it is necessary, knowing $(\boldsymbol{n})$, to calculate the tempered coefficient $\left(\boldsymbol{k}_{\boldsymbol{n}}\right): \quad \boldsymbol{k}_{\boldsymbol{n}}=\sqrt[n_{1}]{2}=1,000000002312091$;

The value of this coefficient with reference to a single scale regulates the total number of frequencies (n) within each spectral range.

Similar processes of the electron incarnation take place in all other $\left(N_{H}-1\right)$ frequency horizons, while the incarnation frequencies of the electron "clones" belonging to the rest of the frequency horizons will be on a common frequency scale in the same allocated band as the frequency $\left(\tilde{\boldsymbol{v}}_{\boldsymbol{R} j}\right)$, related to $\mathbf{j}$-th frequency range in the structure of the wave matrix of physical reality.

Accordingly, each allowed structural frequency $\left(\tilde{\boldsymbol{v}}_{\boldsymbol{R} j}\right)$, in the j-th spectral range, has its own "clone" $\left(\tilde{\boldsymbol{v}}_{H R j}\right)$ in the rest $\left(\boldsymbol{N}_{\boldsymbol{H}}-1\right)$ frequency horizons. Accordingly, in any spectral range of the wave matrix ( $\left.H_{F R e a l}\right)$ in projection onto a single frequency scale, the total number of structural frequencies of physical reality will be represented by the product of the number of frequency horizons by the number of allowed structural frequencies. That is, the tempering coefficient $(\mathrm{kn})$ determines the total number of frequencies in the spectral range involved in all frequency horizons, while the number of frequency horizons $\left(\boldsymbol{N}_{\boldsymbol{H}}\right)$ itself equal to the number of allowed frequencies in a certain spectral range is regulated by the tempering coefficient $-\boldsymbol{k}_{\boldsymbol{H}}$ :

$$
\boldsymbol{k}_{\boldsymbol{H}}=\sqrt[N_{H}]{2}
$$

In the diagram (Fig. 1), the frequency scale $\tilde{\boldsymbol{v}}\left(\boldsymbol{k}_{\boldsymbol{H}}\right)$, related to the wave matrix: $\boldsymbol{A} \tilde{\boldsymbol{v}}=\left(\tilde{\boldsymbol{v}}_{i j}\right)_{\boldsymbol{n}} \boldsymbol{u}$ physical reality is separated from the general frequency scale of the 5 th dimension.

Thus, for the copy-incarnation frequency, the spectrum width $\left(\Delta \tilde{v}_{R j}\right)$ can be calculated on the one hand using formula (12-4), as the difference between two adjacent frequencies in the $\mathrm{j}$-th range, on the other hand, as the sum of the spectral bands $\left(\boldsymbol{\Delta} \tilde{\boldsymbol{v}}_{\boldsymbol{H} \boldsymbol{K} j}\right)$ of all frequencies $\left(\tilde{\boldsymbol{v}}_{\boldsymbol{H} \boldsymbol{K} j}\right)$ considering all frequency horizons. It should be borne in mind that the process of changing the state of a 4dimensional object is synchronized with the process of changing the state of the same object in the 5 th dimension, that is, the number of horizons should be a multiple of a degree of number 2 .

Now, to calculate the number of frequency horizons $\left(N_{H}\right)$, it will be possible to create two equations:

1. $\Delta \tilde{v}_{R j}=\tilde{v}_{R j}\left(k_{H}-1\right)$;

2. $\Delta \tilde{v}_{R j}=\tilde{v}_{H R j}\left(k_{n}-1\right)+\tilde{v}_{H R j}\left(k_{n}-1\right) \cdot k_{n}+\tilde{v}_{H R j}\left(k_{n}-1\right) \cdot k_{n}^{2}+\ldots+\tilde{v}_{H R j}\left(k_{n}-1\right) \cdot k_{n}^{\left(N_{H}-1\right)}(13-5)$ 
Let's simplify expression (13-5):

$$
\Delta \tilde{v}_{R j}=\tilde{v}_{H R j}\left(k_{n}-1\right)\left(1+k_{n}+k_{n}^{2}+\ldots+k_{n}^{N_{H}-1}\right)
$$

In equality (13-6), the entire sum in brackets can be equated to $\boldsymbol{N}_{\boldsymbol{H}}$, then at $\boldsymbol{k}_{\boldsymbol{n}}=1.000000002312$ the equality will take on the final form:

$$
\boldsymbol{\Delta} \tilde{\boldsymbol{v}}_{R j}=\tilde{\boldsymbol{v}}_{H R j}\left(\boldsymbol{k}_{\boldsymbol{n}}-1\right) \cdot N_{H}
$$

Combining both equations, we get:

$$
\tilde{\boldsymbol{v}}_{\boldsymbol{R} j}\left(\boldsymbol{k}_{\boldsymbol{H}}-1\right)=\tilde{\boldsymbol{v}}_{H \boldsymbol{k}}\left(\boldsymbol{k}_{\boldsymbol{n}}-1\right) \cdot N_{H}
$$

It is important to note that the incarnation frequency of the electron $\left(\tilde{v}_{R j}\right)$ is a kind of reference point, which serves to tie each of the frequency horizons to a single frequency scale. In this sense, to simplify the task, the frequency $\left(\tilde{v}_{R j}\right)$, can be taken as the initial frequency for the spectral frequency band of copying-incarnation of all frequency horizons, then

$$
\tilde{v}_{H R j}=\tilde{v}_{R j}+\Delta \tilde{v}_{R j}
$$

Accordingly, equality (13-8) can be represented as follows:

$$
\tilde{\boldsymbol{v}}_{R j}\left(\boldsymbol{k}_{H}-1\right)=\left(\tilde{\boldsymbol{v}}_{R j}+\Delta \tilde{\boldsymbol{v}}_{\boldsymbol{R}}\right) \cdot\left(\boldsymbol{k}_{n}-1\right) \cdot N_{H}
$$

Considering that the number of frequency horizons $\boldsymbol{k}_{\boldsymbol{H}}=\sqrt[N_{H}]{2}$, can be determined as:

$$
N_{H}=\frac{\left(\tilde{v}_{R j}+\Delta \tilde{v}_{R j}\right) \cdot\left(\sqrt[N_{H}]{2}-1\right)}{\tilde{v}_{R j} \cdot\left(k_{n}-1\right)}
$$

Substituting the values $\boldsymbol{k}_{\boldsymbol{n}}=1.000000002312091$ into formula (13-11) and taking into account that the value $\left(\Delta \tilde{\boldsymbol{v}}_{\boldsymbol{R} j}\right)$ is small compared to $\left(\tilde{\boldsymbol{v}}_{\boldsymbol{R} j}\right)$, we can use an iterative method to calculate the $\boldsymbol{N}_{\boldsymbol{H}}$ value at which this equality is satisfied:

$$
N_{H}=\frac{\sqrt[N_{H}]{2}-1}{\left(\boldsymbol{k}_{n}-1\right)}=\frac{\left(\frac{17314}{2}-1\right)}{2,31209110^{-9}}=17314
$$

Given the requirement of frequency multiplicity, the closest multiple of a degree of number 2 will be: $N_{\boldsymbol{H}}=\mathbf{1 6 3 8 4}$, then the tempered coefficient, $\boldsymbol{k}_{\boldsymbol{H}}$ :

$$
\boldsymbol{k}_{H}=\sqrt[N_{H}]{2}=\sqrt[16384]{2}=1,0000423072
$$

Now, knowing both tempering coefficients, it becomes possible to calculate any wave matrix frequency, as well as the spectral width of the structural frequencies, including the copyingincarnation frequency.

From (12-2) the frequency of copying-incarnation in the $\mathbf{j}$-th spectral range:

$$
\tilde{\boldsymbol{v}}_{\boldsymbol{R}}=2,48921300000002 \cdot 10^{20}(\mathrm{~Hz}),
$$

The following structural frequency is determined through the coefficient $\left(\boldsymbol{k}_{\boldsymbol{H}}\right)$ :

$$
\tilde{\boldsymbol{v}}_{(\boldsymbol{R}+1) j}=\tilde{\boldsymbol{v}}_{\boldsymbol{R} \boldsymbol{j}} \cdot \boldsymbol{k}_{\boldsymbol{H}}=2,48931831163225 \cdot 10^{20}(\mathrm{~Hz})
$$

Considering that the spectral width for the frequency $\left(\tilde{\boldsymbol{v}}_{\boldsymbol{R} j}\right)$ of copying the incarnation is controlled by the factor kn, the bandwidth for the frequency $\left(\tilde{\boldsymbol{v}}_{R j}\right)$ in the $\mathrm{j}$-th spectral range is:

$$
\boldsymbol{\Delta} \tilde{\boldsymbol{v}}_{\boldsymbol{H} \boldsymbol{R} j}=\tilde{\boldsymbol{v}}_{\boldsymbol{H} \boldsymbol{j} \boldsymbol{j}} \cdot\left(\boldsymbol{k}_{\boldsymbol{n}}-1\right)=0,575728697 \cdot 10^{12}(\mathrm{~Hz})
$$

Here, the frequency $\tilde{\boldsymbol{v}}_{\boldsymbol{R} j}$ corresponds to the frequency $\tilde{\boldsymbol{v}}_{\boldsymbol{H} R \boldsymbol{j}}$ in projection onto a single frequency scale.

$$
\boldsymbol{k}_{\boldsymbol{n}}=1,000000002312091 \text { (see formula 13-2). }
$$


It should be noted that, in accordance with the principle of separation of structural frequencies, each subsequent frequency $\left(\tilde{v}_{H R j}\right)$ in the sequence of wave matrices is shifted in the cyclic of copyingincarnation process relative to the previous frequency by an amount $\left(\Delta \tilde{\boldsymbol{v}}_{\boldsymbol{H} \boldsymbol{R} j}\right)$ together with the entire wave matrix of the frequency horizon. Moreover, the spectral energy density of vibrations of each subsequent spectral range will be higher than the previous one. The magnitude of this displacement will characterize the angle of inclination of the evolutionary spiral $\left(\boldsymbol{\delta}_{\boldsymbol{e}}\right)$, which is also reflected in the diagram, Fig. 1.

An important stage in our research is the search among previously conducted experiments for experimental data that testify to the reliability of the results obtained. At the same time, taking into account the fundamental nature of the proposals put forward regarding the structure of space, this confirmation should be sought among correctly conducted experiments devoted to the study of the processes of the birth of matter.

Obviously, the existence of another explanation that differs from the generally accepted one in interpreting the results of a particular experiment does not make the experiment itself less significant, requiring a mandatory revision of the results themselves. The repetition of some experiments using modern methods cannot be ruled out. In this case, it is important that the preparation and substantiation of the experimental technique, as well as the interpretation of its results, are carried out in accordance with the provisions of the Theory of the phase medium.

An example of using such an approach is the Sagnac effect, which classical physics has been unsuccessfully trying to explain for more than a hundred years, while the existence of the effect has been repeatedly confirmed at different times by independent researchers, and laser gyroscopes that use this effect have long been used in practice.

According to the formulation proposed by the authors, when the interferometer rotates, the mutual phase shift between two counterpropagating waves is explained by the quantum nature of the process of propagation of electromagnetic waves and arises from the difference in the number of incarnations $\left(\boldsymbol{\Delta} \boldsymbol{P}_{\text {inc }}\right)$ between the waves [1]. That is, the explanation presented simultaneously satisfies both the Sagnac formula and the provisions of the Theory of the phase medium.

The given substantiation and use of the concept: the number of incarnations, expressed in pixels (Pinc), allows us to speak not only about the quantum nature of the process of propagation of electromagnetic waves, but also about the quantum nature of the existence of the wave itself in the phase medium. That is, the longitudinal wave is initially an object in the form of separate discrete states of the phase medium, which is completely renewed with the frequency of incarnations, existing in the structure of spatial frequencies in the form of alternating sections of compression extension. This state of the program level of physical reality can be conventionally represented as a "flickering Hologram".

Then, at the level of the fine structure, the spatio-temporal quantum can be defined as a pixel ( $\boldsymbol{P}_{\text {inc }}$ ), which at the object level in the time domain will be represented as a time quantum, and at the program level (in the frequency plane) the same pixel will be represented by the deformation energy of structural elements phase medium, that is, as a quantum of energy.

It is obvious that any cyclic process in an elastic medium will always be associated with energy losses. Therefore, a dynamic structure, in the form of a quantum of matter (quantum of energy), constantly renewing itself in the cycle of copying-incarnation, can exist only if losses are compensated. 
That is, provided that the energy of the angular momentum is conserved with each new incarnation of the object. As you know, the quality factor " $Q$ " of any resonant system is determined by the ratio of the stored energy to the dissipated power, that is, to the power loss. Therefore, the value of the required energy in the form of an additional impulse will be determined by the elasticity of the medium and can be expressed in terms of the tangent of the loss angle $\left(\boldsymbol{t g} \boldsymbol{\delta}_{\boldsymbol{e}}\right)$. Then the very angle of inclination of the evolutionary spiral $\left(\delta_{e}\right)$, shown in the diagram, Fig. 1, will characterize the rate of change in the state of the object, that is, the rate of the flow of Time.

Simultaneously presented in the diagram, Fig. 1, the model of the process of changing the state of an object allows to draw a parallel between Time and Energy. However, while using the concept of time, not in the previous understanding, as a measure of the duration of processes, but Time in the form of a fraction of the energy spent on renewing a material object in a cyclic process copyingincarnation.

Thus, the angle of inclination of the evolutionary spiral, expressed through the tangent of the angle of losses, acquires the status of a constant that can be defined as a constant of renewable Energy, denoting it as $\boldsymbol{t} \boldsymbol{g} \boldsymbol{\delta}_{\boldsymbol{e}}$.

The tangent of the loss angle can also be represented as the loss factor $\left(\sigma_{e}\right): \sigma_{e}=\boldsymbol{t g} \boldsymbol{\delta}_{\boldsymbol{e}}$; in this case, it will characterize the elastic properties of the phase medium. In this case, the reciprocal of this coefficient will correspond to the $\boldsymbol{Q}$-factor of the phase medium:

$$
Q=\frac{1}{\operatorname{tg} \delta_{e}}
$$

The previously presented tilt angle $\left(\boldsymbol{\delta}_{\boldsymbol{e}}\right)$ characterizing the displacement of the energy spectrum of the wave matrix along the evolutionary spiral can be determined through the tangent of the angle as the ratio of the spectral frequency band $\left(\Delta \tilde{\boldsymbol{v}}_{\boldsymbol{H} R j}\right)$ allocated for the copy-incarnation frequency to the spectral band $\left(\Delta \tilde{v}_{H j}\right)$, allocated for the same frequency taking into account all frequency horizons. Then the tangent of the loss angle:

$$
\operatorname{tg} \delta_{e}=\frac{\Delta \tilde{\boldsymbol{v}}_{H R j}}{\Delta \tilde{\boldsymbol{v}}_{\boldsymbol{H} j}}=\frac{\left(\boldsymbol{k}_{\boldsymbol{n}}-1\right)}{\left(\boldsymbol{k}_{\boldsymbol{H}}-1\right)}=\frac{2,31209 \cdot 10^{-9}}{4,23072 \cdot 10^{-5}}=0,5465005 \cdot 10^{-4}
$$

Here you should pay attention to the fact that the value of tan $\delta$ e with an error of not more than $2.5 \%$ practically coincides with the square of the fine structure constant, which is difficult to explain by simple coincidence, that is: $\boldsymbol{t} \boldsymbol{g} \boldsymbol{\delta}_{\boldsymbol{e}} \equiv(\boldsymbol{\alpha})^{2}$.

Currently, the value of the fine structure constant $(\alpha)$ is determined with a large accuracy and its value: $\boldsymbol{\alpha}=7,2973525693 \cdot 10^{-3}$, respectively: $\boldsymbol{\alpha}^{2}=0,5325135 \cdot 10^{-4}$.

\section{Conclusions}

Thus, the concept of Space-Time Quantum can be substantiated not only from the point of view of a physical model, but also has a quantitative estimate in the form of a fraction of the energy of the geometric field of spatial frequencies spent on updating a material object in the cyclic process of copying-incarnation in the structure of the phase medium.

In other words, the presented fundamental constant equal to the square of the fine structure constant, being expressed in radians through the tangent of the loss angle, determines the angle of inclination of the evolutionary spiral, and thereby regulates the period of state change and the duration of events or processes, that is, it characterizes the rate of the passage of Time. 
As a rule, in such cases, the result of theoretical research requires either experimental confirmation, or multiple rechecking using different calculation methods, or an integrated approach involving the simultaneous participation of both theoreticians and experimenters in research.

The authors hope that a number of independent researchers will respond to the offer to participate in this kind of research.

As you know, the fine structure constant $(\boldsymbol{\alpha})$ was proposed to be introduced by the German physicist Arnold Sommerfeld in 1916. Sommerfeld associated this constant with the physical mechanism of periodic changes in the speed of an electron during its rotation in a circular orbit, which led to the splitting of the energy levels of the atom and the formation of a fine structure in its spectral lines.

Subsequently, researchers were inclined to interpret the fine structure constant as the ratio of two energies, since the small change in the value of the energy levels of the atom recorded in the experiments of Sommerfeld in the form of a set of narrow and close frequencies in its spectral lines turned out to be proportional to $\left(\boldsymbol{\alpha}^{2}\right)$.

One of the versions of the official explanation of the effect is the version according to which the splitting occurs due to the quantum effect of the interaction of two electrons of the atom, as a result of the exchange between them of virtual (unobservable) photons, which leads to a change in the energy of the electron.

Within the framework of the considered model, it can be said that the electron exists simultaneously, as it were, in two frequency ranges - one of which corresponds to the frequency series and the wave matrix: $\boldsymbol{A} \tilde{\boldsymbol{v}}=\left(\tilde{\boldsymbol{v}}_{i j}\right)_{\boldsymbol{n}} \boldsymbol{u}$, based on the frequency of copying-embodiment of physical reality, and the other belongs to a set of independent frequency series of the 5 th dimension.

That is, the effect discovered by Sommerfeld is a kind of analogue of the Doppler Effect, with the only difference that the detected change in the duration of the period relative to the reference frequency is associated not with the movement of the electron and a change in its linear velocity (as Sommerfeld himself interpreted), but with a direct change in the frequency in the process of its renewal and with the replacement of an electron by a clone from the adjacent frequency horizon.

That is, the process of the embodiment of an electron and the materialization of its wave matrix are carried out in two stages, each of which has its own characteristic frequency. First, at the program level, the former copy of the matrix is replaced by a clone of the matrix from the neighboring frequency horizon, then in the second phase - the new copy is embodied in the form of a new state of the electron.

Each cycle of updating the state of a space-time quantum while maintaining its position in spacefrequency coordinates is the rate of Time flow in the conventional sense, that is, as a measure that characterizes the rate of change of states or the duration of events and processes.

Thus, the effect discovered by Sommerfeld is a direct visualization of the tempo of the passage of Time. Moreover, in this sense, the constant of the fine structure must certainly be present and regulate all physical processes in the world of Physical Reality. 
The described research methodology and the results of calculations, as well as the data of experiments carried out at different times by independent researchers, allow, on the basis of the totality of available knowledge and facts, to present the formula for the Scientific Discovery of the physical essence of Time.

\section{Formula of scientific Discovery}

A previously unknown concept has been established that changes the existing concept of the properties of time and determines the physical essence of Time as a fraction of the electromagnetic energy spent on updating a material object in a cyclic process, copying - embodiment in the structure of the phase environment. The value of this fraction, presented as the tangent of the loss angle, is a fundamental constant for all structural levels of physical reality and, being presented in radians, determines the angle of inclination of the evolutionary spiral, thereby regulating the period of state change, the duration of events or processes. The value of this constant can be calculated from the experimental results, and its value turns out to be identically equal to the square of the fine structure constant $\left(\boldsymbol{\alpha}^{2}\right)$. Thus, the effect discovered by Sommerfeld is a direct visualization of the tempo of the Time_flow's.

\section{References}

[1]. Igor Maydykovskiy, Petras Užpelkis: Sagnac effect - experimental confirmation of the Theory of Phase Medium. // Intellectual Archive, 2019, pp. 12 https://doi.org/10.32370/IAJ.2204

[2]. Igor Maydykovskiy, Petras Užpelkis: Holographic principle as the Basis for the Existence of Physical Reality // Intellectual Archive, 2020, pp. 16 https://doi.org/10.32370/IAJ.2348

[3] Igor Maydykovskiy, Petras Užpelkis: Propagation of electromagnetic waves in a phase medium // Intellectual Archive, 2020, pp. 11 https://doi.org/10.32370/IAJ.2383

[4] Latham Boyle, Kieran Finn, and Neil Turok: CPT-Symmetric Universe. // Phys. Rev. Lett. 121, 251301 - Published 20 December 2018 https://doi.org/10.1103/PhysRevLett.121.251301

[5] M. Lachieze-Rey; J. P. Luminet: Cosmic Topology. // Physics Reports (1995) 254 (3), pp: 135214. 